\title{
A protocol for the retina surgeon's safe initial intravitreal injections
}

This article was published in the following Dove Press journal:

Clinical Ophthalmology

9 November 2010

Number of times this article has been viewed

\section{Ronald EP Frenkel ${ }^{1,2}$ \\ Shamim A Haji ${ }^{1,2}$ \\ Melvin La' \\ Max PC Frenkel' \\ Angela Reyes'}

'Eye Research Foundation, Stuart, FL, USA; ${ }^{2}$ East Florida Eye Institute,

Stuart, FL, USA
Correspondence: Ronald Frenkel 509 SE Riverside Dr, Suite 302, Stuart, FL 34994, USA

$\mathrm{Tel}+\mathrm{I} 7722879000$

Fax +I 7722870507

Email efleye@aol.com
Purpose: To determine the safety of a surgeon's initial consecutive intravitreal injections using a specific protocol and to review the complications that may be attributed to the injection procedure. Design: A retrospective chart review.

Participants: Fifty-nine patients (30 females, 29 males) received intravitreal injections of pegaptanib, bevacizumab, or ranibizumab as part of their treatment for neovascular age-related macular degeneration. The average patient age was 80 years. Twenty-two patients were diagnosed with or suspected of having glaucoma. Each patient received an average of 5.8 injections.

Methods: The charts of 59 patients who received a total of 345 intravitreal injections (104 pegaptanib, 74 bevacizumab, 167 ranibizumab) were reviewed. All injections were performed in an office-based setting. Povidone-iodine, topical antibiotics, and eye speculum were used as part of the pre injection procedure. Vision and intraocular pressure were evaluated immediately following each injection.

Main outcome measures: Incidence of post injection complications, including but not limited to endophthalmitis, retinal detachment, traumatic cataract, and vitreous hemorrhage.

Results: There were no cases of endophthalmitis, toxic reactions, traumatic cataracts, retinal detachment, or vitreous hemorrhage. There was one case each of lid swelling, transient floaters, retinal pigment epithelial tear, corneal edema, and corneal abrasion. There were five cases of transient no light perception following pegaptanib injections.

Conclusion: The incidence of serious complications was very low for the intravitreal injections given. A surgeon's initial intravitreal injections may be performed with a very high degree of safety using this protocol.

Keywords: intravitreal injection, post injection complications, intraocular disease, age-related macular degeneration, bevacizumab, endophthalmitis, pegaptanib, ranibizumab

Intravitreal (IVT) injection is a frequently used method for the therapeutic management of many intraocular diseases, particularly those affecting the posterior segment of the eye. Already over the past 5 years, the frequency of IVT injections has increased tremendously for both seasoned and newly trained ophthalmologists. In fact, IVT injection has become one of the most common medical procedures performed in the United States. ${ }^{1}$ Approximately 1.75 million Americans aged over 50 years are living with advanced vision-threatening age-related macular degeneration (AMD), and this number is expected to increase $50 \%$ to 2.95 million by $2020 .{ }^{2}$ Although the complication rate and learning curve of other retinal and ophthalmologic procedures have been investigated, ${ }^{3-7}$ those associated with IVT injections has not. Because of the recent precipitous increase in the frequency of these injections by many surgeons it is important to know how safe these are to our patients. Even experienced surgeons who may have not performed large 
numbers of these procedures in the past now are doing so. It is normal for the newly diagnosed patient with exudative AMD to be anxious about having a needle repeatedly placed in their eye to deliver medication, and it is helpful for them to understand how safe these treatments are.

The Eyetech Phase II study had a $4.8 \%$ incidence of retinal detachment (RD) and the VISION (VEGF Inhibition Study in Ocular Neovascularization) Study Year 1 had a 1.3\% incidence of endophthalmitis. ${ }^{8,9}$ Later the PIER (Phase IIIb, Multicenter, Randomized, Double-Masked, Sham InjectionControlled Study of the Efficacy and Safety of Ranibizumab in Subjects with Subfoveal Choroidal Neovascularization [CNV] with or without Classic CNV Secondary to AMD) study had no cases of endophthalmitis or RDs. ${ }^{10}$ Other serious IVT complications can also include iritis/uveitis, intraocular hemorrhage, ocular hypertension, retinal vascular occlusions, cataract, and hypotony. Prevention of retinal complications is important enough that a vitreoretinal surgical simulator has been developed for novice, inexperienced, and trained surgeons in order to minimize the incidence of these. ${ }^{11}$ This begs the question as to whether a surgeon's initial IVT injections are safe. Although complication rates have been reported in clinical trials, these do not shed light on the initial complication rates of individual surgeons nor of their learning curves. Since most patients who receive these injections have neovascular exudative AMD, and the larger published studies are for this disease, we believed it was important to examine complication rates in this patient group. A highly effective treatment for exudative AMD involves the direct injection of anti vascular endothelial growth factor (anti-VEGF) into the vitreous, which inhibits intraocular angiogenesis. Administering anti-VEGF directly into the vitreous not only maximizes intraocular drug levels, but also minimizes the risk of toxicity associated with systemic administration. ${ }^{12}$ Specifically, the anti-VEGF agents pegaptanib sodium (Macugen; Eyetech Pharmaceuticals, New York, NY) and ranibizumab (Lucentis; Genentech, South San Francisco, CA) have been used successfully in the treatment of exudative AMD. ${ }^{8,13}$ Bevacizumab (Avastin; Genentech), another anti-VEGF derived from the same parent molecule as ranibizumab, has also been used successfully as an IVT injection for the treatment of exudative AMD. ${ }^{14}$

The purpose of this study was to analyze the safety of initial consecutive IVT injections and to review the complications that may be attributed to the injection procedure when utilizing a specific protocol. Given the variation in complication rates that have been published for IVT in different time periods, we also wanted to attempt to understand whether complication rates are related to the learning curve of an individual surgeon or whether they are due to modifications of various methodologies associated with the techniques that have evolved.

\section{Method}

A retrospective study of a single inexperienced vitreoretinal surgeon's first 345 IVT injections was performed. All injections were given in the surgeon's office. Patient charts were reviewed to determine if and which complications occurred. Any complications or serious adverse events were noted in the study, including but not limited to endophthalmitis, RD, traumatic cataract, and vitreous hemorrhage. All IVT injections involved the administration of anti-VEGF agents for the treatment of neovascular AMD in 59 patients (30 females and 29 males). The average patient age was 80 years. A total of 27, 33, and 33 patients received 104, 74, and 167 IVT injections of pegaptanib, bevacizumab, and ranibizumab respectively. Bevacizumab was given as $0.05 \mathrm{~mL}(1.25 \mathrm{mg}$ ) IVT injection, with the frequency determined as per the patient's response, ranibizumab as $0.05 \mathrm{~mL}(0.5 \mathrm{mg})$ IVT injection every 4 weeks, and pegaptanib as $0.09 \mathrm{~mL}(0.3 \mathrm{mg})$ IVT injection every 6 weeks. Initial injections used pegaptanib as it was the first of the three agents available. When bevacizumab was shown to be effective, its use was initiated, and when ranibizumab became covered by insurance, both it and bevacizumab were used. Different sized needles were used, including 27, 30, and 32 gauges, and many patients received more than one type of anti-VEGF injection. Pegaptanib came from the manufacturer with an attached $27 \mathrm{G}$ needle which was always used. Both $30 \mathrm{G}$ and $32 \mathrm{G}$ needles were used for bevacizumab and ranibizumab to determine whether one was any more comfortable than the other to the patient. Vision was evaluated immediately following injection. Patients were examined immediately post injection and monthly to monitor and treat their disease and to detect complications.

The procedure for the IVT administration is described in the following section.

\section{IVT injection procedure}

All injections were performed in an office-based setting following this exact procedure. First, the signed informed consent was confirmed; the affected eye was identified, labeled with a colored sticker above it, and verified with each patient. After the pupil was dilated, the patient was reclined. Calming background music is played to reduce patient anxiety. Care is taken to make sure that the patient is comfortable and is positioned so they can breathe normally 
and keep their head still. Most patients can fully recline but some are uncomfortable and are only reclined as far as their comfort permits. If patients are photophobic the room lights are kept dimmed until the procedure is carried out. Topical anesthesia (proparacaine $0.5 \%$ ) and an antibiotic (Zymar ${ }^{\mathrm{TM}}$ ) were applied to the affected eye. The surrounding eye lashes, caruncle, and upper and lower eyelids were swabbed with povidone-iodine $10 \%$, followed by the insertion of a lid speculum. Using sterile technique, preservative-free $4 \%$ lidocaine was applied for 30 seconds utilizing a cotton tip applicator, followed by two drops of sterile Systane ${ }^{\circledR}$ artificial tears to the cornea. This action was repeated 3 times.

During the procedure, if the cornea began to dry, an additional drop of Systane was given. Then, using sterile technique, one drop of sterile $5 \%$ betadine solution was placed in conjunctival cul-de-sac, avoiding the cornea, for a minimum of 30 seconds and the excess was removed with a cotton applicator. The proper injection site was located at 6-7 o'clock of the right eye or at 5-6 o'clock of the left eye. The surgeon donned nonsterile gloves. A sterile caliper was inspected to make sure the tips were not bent and the exact injection site was located. The patient was given a fixation target superonasally with the assists finger or hand. The needle was uncapped, and the tip was kept away from the mouths of the surgeon, assistant, and patient. In pseudophakic or aphakic patients, the injection site was $3.0-3.5 \mathrm{~mm}$ posterior to the limbus, inferotemporally, and the needle was directed toward the center of the vitreous cavity to a depth of 4-6 mm. In phakic patients, the injection site was 3.5-4.0 mm posterior to the limbus, inferotemporally, and the needle was directed more obliquely half way between the center of the vitreous cavity and the direction of the optic nerve, to a depth of $4 \mathrm{~mm}$. The surgeon then injected $0.09 \mathrm{~mL}$ of pegaptanib using a 27 gauge needle, or $0.05 \mathrm{~mL}$ of ranibizumab, or $0.05 \mathrm{~mL}$ of bevacizumab at the prepped site using either a 30 gauge or 32 gauge needle, over $0.5-2.0$ seconds. After the needle was then immediately withdrawn, the surgeon checked for retinal perfusion by indirect ophthalmoscopy and ensured that each patient could see light and count fingers. An antibiotic (Zymar or Vigamox) was instilled immediately post procedure. Intraocular pressure (IOP) was measured with a tonometer (Tono-Pen; BioRad, Santa Ana, CA) and recorded. In addition, Ciloxan ointment was applied to the affected eye, and the eye was patched. Each patient was given written post operative instructions and warned of the symptoms of RD and endophthalmitis. Each patient was also given or prescribed an antibiotic (Zymar or Vigamox) to instill at home, 4 times a day for 3 days. Patients were followed for the development of complications for 6 months after the last injection.

\section{Results}

There were no complications of myocardial infarction, stroke, endophthalmitis, toxic reactions, traumatic cataracts, $\mathrm{RD}$, or vitreous hemorrhage (Table 1). One eye developed lid swelling, one eye developed transient floaters, one eye exhibited a retinal pigment epithelial (RPE) tear, one eye developed corneal edema, and one eye sustained a corneal abrasion. Mean preoperative IOP was $13 \mathrm{mmHg}$. All the three IVT injections caused significant initial IOP spikes: mean IOP of $38.5 \pm 11.56 \mathrm{mmHg}$ in the pegaptanib group, $37.75 \pm 8.36 \mathrm{mmHg}$ in the ranibizumab group, and $34.88 \pm 10.45 \mathrm{mmHg}$ in the bevacizumab group. There was no quantitative rate control, and the rate of injection was decided by how likely the surgeon believed the eye or head might move, with a faster rate utilized for those who appeared less steady. The IOP normalized after about 30 minutes post injection in all the patients. This was in concert with our previous findings on IOP effects of IVT injections in patients with and without glaucoma. ${ }^{15}$

There was a $4.8 \%$ (5 out of 104 ) incidence per injection of transient no light perception (NLP) following pegaptanib injections, and all of these patients had immediate post injection IOPs of greater than $55 \mathrm{mmHg}$, while no patients went NLP following bevacizumab or ranibizumab injections. Our surgeon performed an average of 5.8 injections per patient.

\section{Discussion}

In this retrospective analysis, the complication rate of one surgeon's initial consecutive IVT injections compares favorably with published rates in the major clinical trials of ranibizumab

Table I Complications and adverse events in our study (per injection, $\mathrm{n}=345$ )

\begin{tabular}{ll}
\hline Complications & Number of cases \\
\hline Lid swelling & $\mathrm{I}$ \\
Vitreous floaters & $\mathrm{I}$ \\
RPE tear & $\mathrm{I}$ \\
Corneal edema & $\mathrm{I}$ \\
Corneal abrasion & $\mathrm{I}$ \\
Transient NLP & $5^{\mathrm{a}}$ \\
Arterial thrombotic events & 0 \\
Endophthalmitis & 0 \\
Retinal detachment & 0 \\
Traumatic cataract & 0 \\
Vitreous hemorrhage & 0 \\
\hline
\end{tabular}

Note: aPegaptanib group only

Abbreviations: RPE, retinal pigment epithelial; NLP, no light perception. 
and pegaptanib (Table 2). No incidence of endophthalmitis was recorded among the 345 injections performed in our study. Among the ranibizumab clinical trials, the MARINA (Minimally Classic/Occult Trial of the Anti-VEGF Antibody Ranibizumab in the Treatment of Neovascular AMD) study ${ }^{16}$ had a per patient incidence of endophthalmitis of $1.0 \%$ ( 5 cases in 477 patients), while no cases of endophthalmitis were reported in the PIER study. ${ }^{10}$ In the MARINA study, four of the five cases of endophthalmitis were culture negative. For pegaptanib, the VISION study reported an incidence rate of $1.3 \%$ per patient (12 cases in 892 patients). ${ }^{9}$ The Eyetech Phase II study did not specify whether or not they had any cases of endophthalmitis. ${ }^{8}$ Gragoudas et al attributed many of the infections in the VISION study to protocol violations, the most common being the failure to use a lid speculum. ${ }^{17}$ The antisepsis procedure originally described in the VISION study protocols was revised in a protocol amendment after approximately $60 \%$ of the injections were administered for the 2 years of these studies. The amendment reinforced the aseptic nature of the procedure and mandated the use of (1) sterile preparation and drape similar to that used for routine intraocular surgery and (2) either preinjection topical ophthalmic antibiotic drops for 3 days before the injection or a $10-\mathrm{mL}$ povidone-iodine flush immediately before injection.

Improvements in sterile procedure apparently reduced the incidence of endophthalmitis in Year 2 of the VISION study, as none were reported in the second year. It should also be noted that the 27-gauge needle size used for pegaptanib is larger than that used for ranibizumab and bevacizumab IVT injections. The lower incidence of endophthalmitis in our study compared with those reported in the VISION study is likely related to lessons learned since that trial regarding the prevention of endophthalmitis. These include strict attention to sterile technique, including the use of a lid speculum and povidone-iodine, and possibly topical antibiotics both before and after injection. ${ }^{18}$ The use of povidone-iodine is able to reduce the number of ocular surface bacteria by $91 \%$, the likely source of post procedure infection. ${ }^{19}$ The use of topical antibiotics is up to the physician's discretion, but antimicrobial resistance should be considered when selecting an appropriate antibiotic.

Some concern has been brought up over the use of bevacizumab in IVT injections as being another possible source of infection. While pegaptanib and ranibizumab are labeled for IVT use, bevacizumab is labeled for use in cancer therapy and is currently being used "off-label" for the treatment of exudative AMD. Because of its "off-label" use, bevacizumab is supplied in much larger volumes than those needed for single IVT doses. Thus, hospitals and compounding pharmacies must divide the larger volume of bevacizumab into smaller units suitable for single-use, individual doses. Contaminants could possibly be introduced during the compounding process and compromise the sterility of the aliquoted drug. Although we used bevacizumab prepared by a compounding pharmacy, we did not have a higher incidence of endophthalmitis. Our incidence of endophthalmitis still compares favorably with the rates in clinical trials of ranibizumab and pegaptanib.

No incidence of RD was reported in our study nor in the MARINA and PIER studies. ${ }^{10,16}$ The incidence rate of RD was $4.8 \%$ ( 1 case in 21 patients) in the Eyetech study and $0.7 \%$ (6 cases in 892 patients) in the first year of the VISION study. 8,9

Table 2 Complications and adverse events secondary to IVT injections performed by one surgeon, compared with ranibizumab and pegaptanib clinical trials (incidence per patient)

\begin{tabular}{|c|c|c|c|c|c|c|}
\hline Complications & $\begin{array}{l}\text { Our study } \\
n=59\end{array}$ & $\begin{array}{l}\text { Rosenfeld, } \\
\text { MARINA study }{ }^{16} \\
n=477\end{array}$ & $\begin{array}{l}\text { Regillo, } \\
\text { PIER study }{ }^{10} \\
n=120\end{array}$ & $\begin{array}{l}\text { Eyetech study, } \\
\text { Phase } I^{8} \\
n=2 I\end{array}$ & $\begin{array}{l}\text { D'Amico, VISION } \\
\text { study, Year }\left.\right|^{9} \\
n=892\end{array}$ & $\begin{array}{l}\text { D'Amico, VISION } \\
\text { study, Year } 2^{9} \\
n=606\end{array}$ \\
\hline Lid swelling & $1.7 \%$ & $\mathrm{~N} / \mathrm{A}$ & $\mathrm{N} / \mathrm{A}$ & $14.3 \%$ & $N / A$ & $N / A$ \\
\hline Vitreous floaters & $1.7 \%$ & $N / A$ & N/A & $19.0 \%$ & $33 \%$ & $24 \%$ \\
\hline RPE tear & $1.7 \%$ & $0.4 \%$ & 0 & N/A & $N / A$ & $\mathrm{~N} / \mathrm{A}$ \\
\hline Corneal edema & $1.7 \%$ & $N / A$ & N/A & $\mathrm{N} / \mathrm{A}$ & $10 \%$ & $9 \%$ \\
\hline Corneal abrasion & $1.7 \%$ & $\mathrm{~N} / \mathrm{A}$ & $\mathrm{N} / \mathrm{A}$ & $14.3 \%$ & N/A & $\mathrm{N} / \mathrm{A}$ \\
\hline Transient NLP & $8.5 \%$ & N/A & $N / A$ & N/A & $N / A$ & $\mathrm{~N} / \mathrm{A}$ \\
\hline Arterial thrombotic events & 0 & $3.8 \%$ & 0 & $N / A$ & $3 \%$ & $2 \%$ \\
\hline Endophthalmitis & 0 & $1.0 \%$ & 0 & $\mathrm{~N} / \mathrm{A}$ & $1.3 \%$ & $0.6 \%$ \\
\hline Retinal detachment & 0 & 0 & 0 & $4.8 \%$ & $0.7 \%$ & $1.2 \%$ \\
\hline Traumatic cataract & 0 & $0.2 \%$ & 0 & 0 & $0.6 \%$ & $0.2 \%$ \\
\hline Vitreous hemorrhage & 0 & $0.4 \%$ & 0 & $\mathrm{~N} / \mathrm{A}$ & $1.8 \%$ & $1.8 \%$ \\
\hline
\end{tabular}

Abbreviations: IVT, intravitreal; RPE, retinal pigment epithelial; N/A, not applicable; NLP, no light perception. 
Out of six cases of RD reported in the VISION study, two subjects had RDs that were exudative/hemorrhagic in nature and were attributed to the underlying disease process. The other four subjects had RDs with a rhegmatogenous component; risk factors included retinoschisis in the study eye of one of these subjects, and lattice degeneration in the study eye and a history of RD in the fellow eye in a second subject. We believe that indirect ophthalmoscopy to examine the peripheral retina prior to the IVT injection is necessary to help avoid such complications in high-risk patients like high myopia with lattice degeneration, history of retinoschsis, and any previous history of RD in fellow eye. No cases of vitreous hemorrhage or lens damage (traumatic cataract) were reported in our study. The incidence of these complications was rare in the MARINA study, with only two cases of vitreous hemorrhage and one case of traumatic cataract being reported out of 477 patients. ${ }^{16}$ No cases of traumatic cataract or vitreous hemorrhage were reported in the PIER study. ${ }^{10}$ In the first year of the VISION study, the per patient incidence of vitreous hemorrhage was $1.8 \%$ (16 cases/892 patients). ${ }^{9}$ It was determined that the hemorrhage in 7 of the 16 subjects was related to underlying choroidal neovascularization, while the other nine cases were related to the injection procedure. The incidence of traumatic cataract was $0.6 \%$ and $0.2 \%$ in the first and second year, respectively. Many cases of traumatic cataract can be attributed to the injection procedure, where the needle contacts or penetrates the lens capsule. ${ }^{20}$ However, these complications are rare, both in our study and in the clinical trials shown here. We believe measuring distance from limbus with a caliper $3.5-4.0 \mathrm{~mm}$ posterior to the limbus in phakic eyes versus $3.0-3.5 \mathrm{~mm}$ posterior to the limbus in pseudophakic eyes reduced the incidence of iatrogenic cataract formation in our group. Fine-tipped calipers can become bent with use, defeating their purpose, so they must be inspected.

Other adverse events recorded in our study include one case each of lid swelling, RPE tear, corneal edema, corneal abrasion, and transient vitreous floaters. The lid swelling occurred within one day of the procedure and was due to a local reaction to the pre injection surgical preparation. We now know that RPE tears are more likely to occur in patients with large pigment epithelial detachments who receive anti-VEGF injections, and this was true of our patient who developed this within one month of the treatment, but this can be part of the natural history of exudative AMD as well. ${ }^{21}$

Perhaps such patients should receive lower doses of antiVEGF drugs, but there is no current evidence to support this. The cause of the corneal edema which was evident within
1 week postoperatively was unclear, but it resolved with time. The corneal abrasion is believed to be due to the drying of the corneal epithelium due to the patient being unable to blink while a lid speculum was in place. Not all clinical trials made note of these complications. In the MARINA study, two cases of RPE tear $(0.4 \%$ per patient $)$ were recorded. ${ }^{16}$ In the VISION study, the incidence of vitreous floaters was more common, occurring in 33\% of the study participants. ${ }^{9}$ Corneal edema occurred in $10 \%$ of the patients in the VISION study. The Eyetech study noted lid swelling in $14.3 \%$ of their patients, vitreous floaters in $19.0 \%$, and corneal abrasion in $14.3 \% .{ }^{8}$ The occurrences of vitreous floaters in the VISION and Eyetech studies were transient in nature and attributed to the injection procedure. Our patient noted this within one day of the procedure.

We believe that low incidence of corneal edema and corneal abrasion in our study $(1.7 \%$ and $1.7 \%$ versus $9 \%-10 \%$ and $14.3 \%$ respectively) was because of the use of preservative-free, single unit $4 \%$ lidocaine and the judicious use of artificial tears. After the initial corneal abrasion, we learned the importance of avoiding drying of the corneal epithelium. The cornea must be kept moist particularly in patients with a preexisting abnormal corneal surface such as those with dry eyes and those who frequently instill drops with preservatives such as glaucoma patients on multiple medications.

Five cases in our study noticed transient NLP immediately post injection. All five case received pegaptanib, which is a larger $0.09 \mathrm{~mL}$ volume as compared with $0.05 \mathrm{~mL}$ of bevacizumab and ranibizumab. We believe that the transient NLP in the petaptanib group was due to a volume-mediated IOP spike causing transient ischemia. All these patients underwent immediate anterior chamber paracentesis, which normalized the IOP. Although smaller volumes of anti-VEGF are being used now, we thought it may be relevant to discuss this from a historical standpoint. Information on short-term IOP trends after IVT injections is limited; particularly in patients with glaucoma as clinical trials on antivascular endothelial growth factor therapies tend to exclude eyes with glaucoma. The effect of the IOP spikes on the already compromised optic nerve in glaucoma patients is not known. However, caution may be prudent in patients with advanced glaucoma, and pre-injection paracentesis may be considered in patients who have demonstrated significant IOP spikes after previous injections, when repeated injections are indicated. We don't currently recommend doing a paracentesis in all patients who develop transient NLP as we did in the patients in this study. Needle size did not correlate with complications other than 
the association of the larger needle with the higher IOP and transient NLP in the pegaptanib group, but it does not appear to be causative. However, we did not use a $27 \mathrm{G}$ needle with the other medication volumes so we cannot state that with certainty. There were no other types of complication associated with the IOP spikes.

Although a formal analysis was not performed, it did not seem as though there was a real difference in comfort level between the $30 \mathrm{G}$ versus $32 \mathrm{G}$ needle, though both were more comfortable than the $27 \mathrm{G}$ needle.

Although the numbers of patients in the clinical studies were in general larger, we believe that the initial 345 injections performed by a single surgeon provides an excellent assessment of the "learning curve" associated with this procedure. This is an important issue given the frequency of IVT injections, the recent dramatic increase in their frequency, and the potential complications. As our study has shown, initial injections can be performed with an excellent safety profile even during the initial stage of a physician's experience with IVT injections. The transient cases of NLP vision were unrelated to the "learning curve" based on their sporadic occurrence.

There are some obvious limitations to this study. This is a retrospective investigation of a single surgeon's IVT injections, without randomized comparisons between alternate techniques or other treating ophthalmologists. Also, our study included patients who received IVT for exudative AMD only. The surgeon had very limited experience, giving less than 10 IVT injections for causes other than AMD that were not part of this study. Though these occurred during this same time period, they did not affect the conclusions of this study and were not associated with complications other than the known effect of increased IOP after a Kenalog injection.

We learned that IVT is safe to perform when: the patient is comfortably positioned so they may remain still; proper sterilization techniques are utilized, including 5\% betadine; calipers are used to measure the exact injection site (to avoid iatrogenic cataract formation and retinal breaks); those patients with a history of high myopia, lattice degeneration, retinoschisis, or a previous history of $\mathrm{RD}$ are followed closely; the cornea is kept moist at all times with the use of artificial tears; and preservative-free lidocaine is utilized during the injection procedure. Though the results of others may vary, we have shown in this study that a surgeon's initial IVT injections with this protocol may be performed with a high degree of safety in the office setting, and with a very low risk of ocular complications. We believe that if our procedure is meticulously followed, complications will be reduced and patients will be even more accepting of the treatment. Given the precipitous increase in the frequency of these injections, this is encouraging to all of our patients.

\section{Note}

This work was presented in part at the Association for Research in Vision and Ophthalmology (ARVO) Annual Meeting 2008.

\section{Disclosure}

The authors report no conflicts of interest in this work.

\section{References}

1. Centers for Medicare and Medicaid Services. Medicare Utilization for Part B. [cited 2008 Aug 16]. Available from: http://www.cms.hhs. gov/MedicareFeeforSvcPartsAB/04_MedicareUtilizationforPartB.asp. Accessed 2010 Oct 2.

2. Eye Diseases Prevalence Research Group. Prevalence of age related macular degeneration in the United States. Arch of Ophthalmol. 2004;122:564-572.

3. Gopal L, Sharma T, Shanmugam M, et al. Surgery for stage 5 retinopathy of prematurity: the learning curve and evolving technique. Indian J Ophthalmol. 2000;48(2):101-106.

4. Lee J, Hou C, Yang M, Kuo JZ, Lin K. A different approach to assess resident phacoemulsification learning curve: analysis of both completion and complication rates. Eye. 2009;23(3):683-687.

5. Al-Swailem SA, Wagoner MD. Complications and visual outcome of LASIK performed by anterior segment fellows vs experienced faculty supervisors. Am J Ophthalmol. 2006;141(1):13-23.

6. Yildirim R, Devranoglu K, Ozdamar A, Aras C, Ozkiris A, Ozkan S. Flap complications in our learning curve of laser in situ keratomileusis using the Hansatome microkeratome. Eur J Ophthalmol. 2001;11(4): $328-332$.

7. Randleman JB, Wolfe JD, Woodward M, Lynn MJ, Cherwek DH, Srivastava SK. The resident surgeon phacoemulsification learning curve. Arch Ophthalmol. 2007;125(9):1215-1219.

8. Eyetech Study Group. Anti-vascular endothelial growth factor therapy for subfoveal choroidal neovascularization secondary to age-related macular degeneration: Phase II study results. Ophthalmology. 2003; 110(5):979-986.

9. D'Amico DJ, Masonson HN, Patel M, et al; for the VEGF Inhibition Study in Ocular Neovascularization (V.I.S.I.O.N.) Clinical Trial Group. Pegaptanib sodium for neovascular age-related macular degeneration: two-year safety results of the two prospective, multicenter, controlled clinical trials. Ophthalmology. 2006;113(6):992-1001.

10. Regillo CD, Brown DM, Abraham P, et al. Randomized, double-masked, sham-controlled trial of ranibizumab for neovascular age-related macular degeneration: PIER Study Year 1. Am J Ophthalmol. 2008; 145(2):239-248.

11. Rossi JV, Verma D, Fujii GY, et al. Virtual vitreoretinal surgical simulator as a training tool. Retina. 2004;24(2):231-236.

12. Csaky DK. Anti-vascular endothelial growth factor therapy for neovascular age-related macular degeneration: promises and pitfalls. Ophthalmology. 2003;10(5):879-881.

13. Schmidt-Erfurth UM, Pruente C. Management of neovascular agerelated macular degeneration. Prog Retin Eye Res. 2007;26(4): $437-451$. 
14. Levy J, Shneck M, Rosen S, et al. Intravitreal bevacizumab (avastin) for subfoveal neovascular age-related macular degeneration. Int Ophthalmol. 2010; DOI: s10792-008-9243-1. Accessed 2010 Oct 2.

15. Frenkel REP, Mani L, Toler AR, Frenkel MPC. Intraocular pressure effects of pegaptanib (Macugen) injections in patients with and without glaucoma. Am J Ophthalmol. 2007;143(6):1034-1035.

16. Rosenfeld PJ, Brown DM, Heier JS, et al; and the MARINA Study Group. Ranibizumab for neovascular age-related macular degeneration. N Engl J Med. 2006;355(14):1419-1431.

17. Gragoudas ES, Adamis AP, Cunningham ET, Feinsod M, Guyer DR; for the VEGF Inhibition Study in Ocular Neovascularization (VISION) Clinical Trial Group. Pegaptanib for neovascular age-related macular degeneration. N Engl J Med. 2004;351(27):2805-2816.
18. Aiello LP, Brucker AJ, Chang S, et al. Evolving guidelines for intravitreous injections. Retina. 2004;24(5):S3-S19.

19. Apt L, Isenberg S, Yoshimori R, Paez J. Chemical preparation of the eye in ophthalmic surgery III: effect of povidone-iodine on the conjunctiva. Arch Ophthalmol. 1984;102:728-729.

20. Jager RD, Aiello LP, Patel SC, et al. Risks of intravitreous injection: a comprehensive review. Retina. 2004;24(5):676-698.

21. Chan CK, Abraham P, Meyer CH, et al. Optical coherence tomography-measured pigment epithelial detachment height as a predictor for retinal pigment epithelial tears associated with intravitreal bevacizumab injections. Retina. 2010;30(2):203-211.
Clinical Ophthalmology

\section{Publish your work in this journal}

Clinical Ophthalmology is an international, peer-reviewed journa covering all subspecialties within ophthalmology. Key topics include: Optometry; Visual science; Pharmacology and drug therapy in eye diseases; Basic Sciences; Primary and Secondary eye care; Patien Safety and Quality of Care Improvements. This journal is indexed on

Submit your manuscript here: http://www.dovepress.com/clinical-ophthalmology-journal

\section{Dovepress}

PubMed Central and CAS, and is the official journal of The Society of Clinical Ophthalmology (SCO). The manuscript management system is completely online and includes a very quick and fair peer-review system, which is all easy to use. Visit http://www.dovepress.com/ testimonials.php to read real quotes from published authors. 